The Astrophysical Journal, Vol. 156, April 1969

(C) 1969. The University of Chicago. All rights reserved. Printed in U.S.A.

\title{
INFRARED OBSERVATIONS OF ETA CARINAE TO 20 MICRONS*
}

\author{
J. A. Westphal and G. Neugebauer \\ California Institute of Technology, Pasadena, and Cerro Tololo Inter-American Observatory $\dagger$ \\ Received February 28, 1969
}

\section{ABSTRACT}

Measurements of $\eta$ Carinae have been obtained at 4.8,10.1, and $19.5 \mu$ which show a continued increase in flux density into the infrared. The bolometric magnitude is estimated to be -12 .

In 1967 infrared observations of the non-stellar object $\eta$ Carinae showed that its continuum radiation increased strongly up to wavelengths of $3.4 \mu$ (Neugebauer and Westphal 1968; hereinafter referred to as "Letter I"). In the present Letter observations which extend measurements of the continuum of $\eta$ Car to $19.5 \mu$ are given.

Photometric measurements of $\eta$ Car at effective wavelengths of 4.8,10.1, and $19.5 \mu$, with $20^{\prime \prime}$ apertures, are shown in Figure 1 along with the data of Letter I and those of Rodgers and Searle (1967). The new infrared data were obtained between July 2 and 16, 1968, with the 60-inch telescope of the Cerro Tololo Inter-American Observatory in Chile. The photometer was the same as that used to make the observations reported in Letter I; however, a liquid-nitrogen-cooled $\mathrm{PbSe}$ cell was used as a detector at $4.8 \mu$, while a $\mathrm{Ge}$ : $\mathrm{Cd}$ cell cooled by liquid hydrogen was used at both 10.1 and $19.5 \mu$. The observed wavelength intervals, defined by interference filters, were $4.5-5.1 \mu\left(\lambda_{\text {eff }}=\right.$ $4.8 \mu), 8.1-13.5 \mu\left(\lambda_{\text {eff }}=10.1 \mu\right)$, and 16.2-23.5 $\mu\left(\lambda_{\text {eff }}=19.5 \mu\right)$.

The magnitude at $4.8 \mu$ was obtained by comparing observations of $a$ Sco, $a$ Her, and R Hya with observations by Johnson (1964) and Becklin (1968); the ratios of the observed intensities corrected to unit air mass were $I(\eta \mathrm{Car}): I(a \mathrm{Sco}): I(a \mathrm{Her}): I(\mathrm{R} \mathrm{Hya})=$ 1.6:1.8:1.4:1.0. The magnitude at $10.1 \mu$ was based on photometry of $a$ Sco and $a$ Her by Low and Johnson (1964): $I(\eta$ Car $): I(a$ Sco $): I(a$ Her $)=46: 1.7: 1.0$. The calibration of the 19.5- $\mu$ data was based on observations of a Sco and satellite IV of Jupiter: $I(\eta \mathrm{Car}): I(\mathrm{~J} \mathrm{IV}): I(a$ Sco $)=100: 1.3: 1.0$. The energy calibrations of the photometry were obtained from Low (1968). For the 10.1- and 19.5- $\mu$ measurements the standards required approximately ten object-sky differences in order to obtain a signal-to-noise ratio of $5: 1$, while a single measurement of $\eta$ Car resulted in a signal-to-noise ratio greater than 100:1. Thus the dominant inaccuracy in this measurement results from the uncertainty in the measurement and absolute calibration of the standard stars. Observations at $4.8 \mu$ were obtained on three nights, at $19.5 \mu$ on two nights, and at $10.1 \mu$ on one night.

Although the data presented in Figure 1 were obtained at different times, and $\eta$ Car is known to vary, there is evidence that the compilation can be taken as representative of the continuum of $\eta$ Car within the errors shown. Measurements of $\eta$ Car at $2.2 \mu$ which were made in 1968 agreed with those made in 1967 to within 5 per cent. Haug (1968) obtained a visual magnitude of $V=6.08, B-V=0.63$, and $U-B=0.34$ mag on

* Contributions from the Cerro Tololo Inter-American Observatory, No. 62.

This work was supported in part by National Science Foundation grant GP 15-25 and National Aeronautics and Space Administration grant NGL 05-002-007.

$\dagger$ Operated by the Association of Universities for Research in Astronomy, Inc., under contract with the National Science Foundation. 
July 5, 1968, which can be compared with the values $V=6.12, B-V=0.7$, and $U-B=0.4 \mathrm{mag}$ obtained by Walker (1967) at the time the 1.6-, 2.2-, and 3.4- $\mu$ measurements were made in 1967. The value $V=6.38$ was derived from the data obtained by Rodgers and Searle (1967).

Figure 2 of Letter I presented data indicating that essentially all of the radiation from $\eta$ Car both at $2.2 \mu$ and in the visible is emitted within an area 15" in diameter, while a corelike emitting region whose diameter is typically $5^{\prime \prime}$ is also indicated. At $4.8 \mu$ apertures ranging from $10^{\prime \prime}$ to $50^{\prime \prime}$ were used; the signal closely followed the data presented in Figure 2 of Letter I. The measurements at 10 and $19.5 \mu$ were made only with a $20^{\prime \prime}$ aperture.

In addition to the photometric observations, low-resolution spectral scans were obtained over the wavelength region 2.0-2.5 $\mu$. These scans were made by adding a circular interference-filter wheel to the photometer; the resolution is 1.5 per cent of the wavelength. Fifty-six spectral scans of $\eta$ Car, each taking 1 minute, were obtained and averaged; this average is presented in Figure 2 of this Letter along with a comparison scan of $a$ Boo. Spectral scans of $\mu \mathrm{Vel}$, whose magnitude at $2.2 \mu$ is more comparable to that of $\eta$ Car, were also taken to check for systematic effects in the averaging techniques. The obvious emission feature at $2.17 \mu$ in the spectrum of $\eta$ Car can be identified with the Brackett 7 emission line of hydrogen. Its equivalent width is $50 \pm 10 \AA$, corresponding to an observed flux in the line of $7.1 \pm 1.5 \times 10^{-13} \mathrm{~W} \mathrm{~m}^{-2}$.

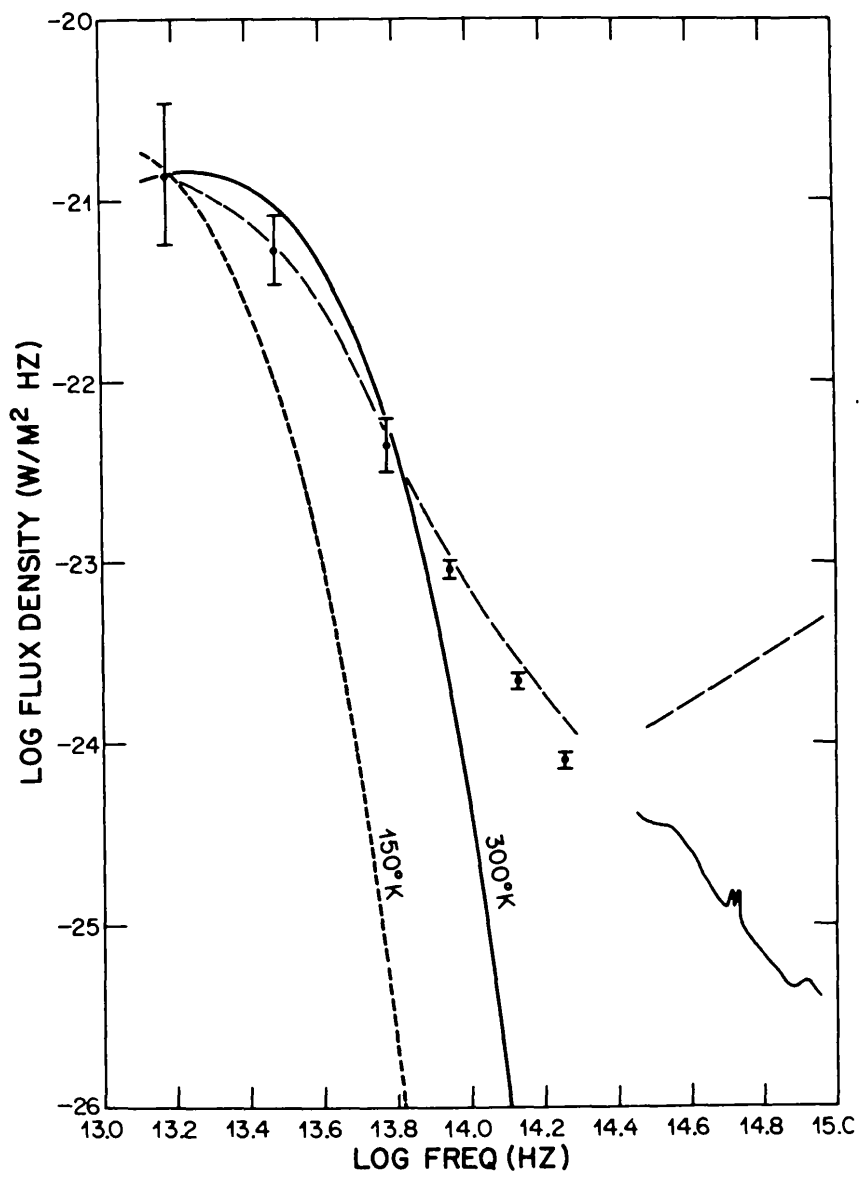

FIG. 1.-Observed infrared photometry of $\eta$ Car, shown as data points; the solid line at high frequencies represents the data of Rodgers and Searle (1967). The dashed line above these points takes into account the reddening as found by Pagel (1969). Calculated curves for blackbody emission at $150^{\circ}$ and $300^{\circ} \mathrm{K}$ are also shown. 
The true continuum spectrum of $\eta$ Car in the visual region must be affected by interstellar extinction. Estimates of the extinction have ranged from a total visual extinction $A_{V} \sim 1$ mag to $A_{V} \sim 2$ mag (Faulkner 1963; Feinstein 1963; Rodgers and Searle 1967). Recently, Pagel (1969) has re-examined the relative intensities of Fe II lines and finds evidence for a value of reddening $E_{B-V}=1.2$ or $A_{V} \sim 3.6$ mag. An estimate of the reddening made by a comparison of the measured B7 line with the Balmer and Paschen line strengths measured by Aller (1966) and Rodgers and Searle (1967) is in agreement with this estimate. As noted by Pagel (1969), this result leads to a rise in the continuum spectrum toward the ultraviolet as well as toward the infrared. The data corrected to account for this reddening are included in Figure 1. A value of 3.6 mag visual absorption will be used in the following discussion.

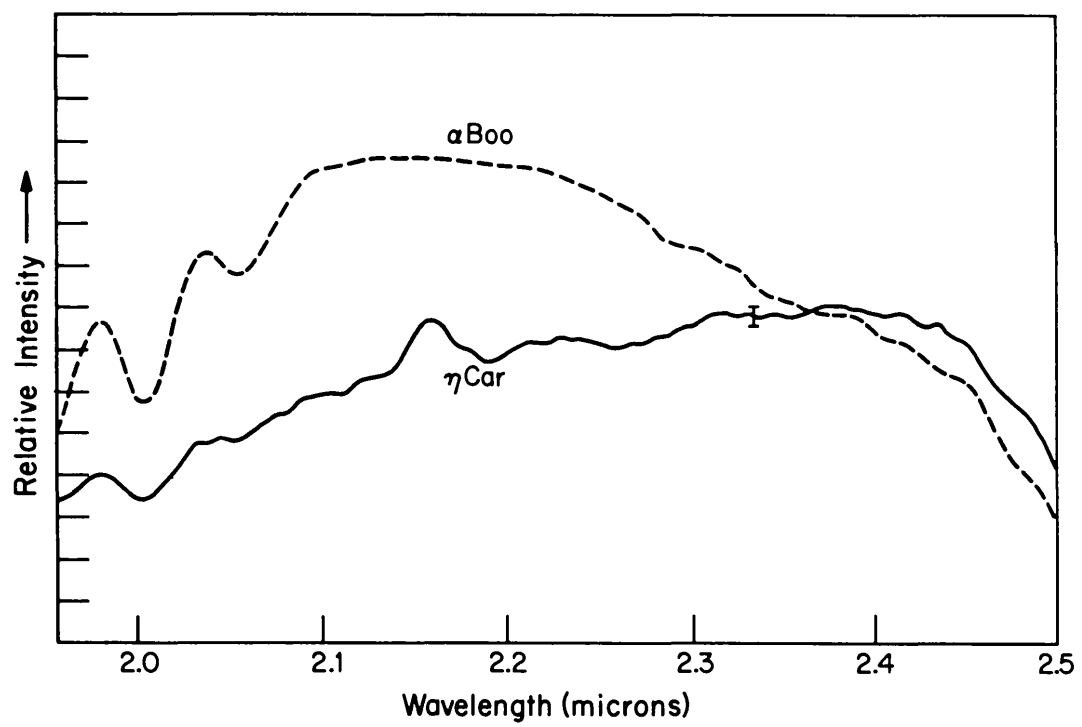

FIG. 2.-Spectra of $\eta$ Car (solid line) and $a$ Boo (dashed line) are shown; the amplifier gain of the $a$ Boo scan is reduced by a factor of 31.6 below that used with the $\eta$ Car data. Errors in the spectrum of $\eta$ Car, which was averaged from fifty-six 1-minute scans, are typified by the error bar shown at $\sim 2.3 \mu$.

\section{DISCUSSION}

The most striking feature of the infrared data is the strength of the continuum of $\eta$ Car in the infrared. With the exception of objects within the solar system, $\eta$ Car is the brightest object at 10 and $20 \mu$ known to the authors.

A calculation of the intrinsic luminosity of $\eta$ Car is complicated by uncertainty in its distance, by the unknown interstellar extinction, and by the unknown nature of the spectrum at longer wavelengths. Estimates of the distance to $\eta$ Car range from 0.6 to $2.8 \mathrm{kpc}$ (Faulkner 1963; Feinstein 1963), but most recent determinations favor a value around $1.5 \mathrm{kpc}$ (Gratton 1963). If this value of distance and Pagel's (1969) estimate of the extinction are assumed, the total power emitted between 0.4 and $19.5 \mu$ is approximately $7.5 \times 10^{32} \mathrm{~W}$, or $1.9 \times 10^{6} \mathrm{~L} \odot$; about 90 per cent of this energy is emitted at wavelengths longward of $1.65 \mu$.

If one assumes that the total unobserved continuum energy is greater than that which is observed by a factor of roughly 2 , the emitted radiation corresponds to a bolometric magnitude of -12 . It is interesting to note that the bolometric magnitude has been estimated as having equaled -12.5 in 1843 , when $\eta$ Car reached its maximum visual brightness (Gratton 1963). One is tempted to suggest that this agreement, although perhaps fortuitous, is an indication that the absolute luminosity of $\eta$ Car has remained essentially constant since 1843 and that the energy has been redistributed in wavelength since that time. 
The new photometric data, when incorporated into Pagel's (1969) discussion, strengthen the idea that the continuum at wavelengths longward of $\sim 1.6 \mu$ results from a different mechanism from that responsible for the visual continuum. The large flux density at $20 \mu$ makes it increasingly difficult to reconcile the upper limit of $8 \times 10^{-26}$ $\mathrm{W} \mathrm{m} \mathrm{m}^{-2} \mathrm{~Hz}^{-1}$, observed by Beard and $\mathrm{Kerr}(1967)$ at $11 \mathrm{~cm}$, with a synchrotron origin for the infrared continuum (McCray 1967).

The possibility that the infrared continuum of $\eta$ Car is of thermal origin appears to have been reinforced; this has been discussed by Pagel (1969), among others, and in Letter I. As pointed out previously, however, the continuum cannot be attributed to an optically thick source characterized by a single temperature; blackbody emission curves for representative temperatures are also included in Figure 1.

Although it is clear that recombination radiation is not a major contributor to the observed continuum, it is perhaps worth while to consider the evidence to that effect which can be obtained from the measurement of the B7 emission line. In view of the obvious real complexity of $\eta$ Car, these calculations must be considered as in the realm of numerology. The expected equivalent width of the B7 line, following Seaton (1960), is $200 \AA$ if one considers only free-free and bound-free continuum at an effective temperature of $20000^{\circ} \mathrm{K}$. Thus, from the observed equivalent width of $50 \AA$, one estimates that at $2.16 \mu$ only about one-fourth of the observed continuum is due to free-free and bound-free transitions. An extrapolation of this into the visual region indicates that at $0.56 \mu$ only one-twentieth of the observed continuum is caused by hydrogen recombination.

If one further assumes that the $\mathrm{B} 7$ line arises in a spherical volume with a 2 ".5 radius, the observed line strength implies an electron density of $4 \times 10^{5}$ electrons $\mathrm{cm}^{-3}$. This value is in agreement with the estimate of $2 \times 10^{5}$ electrons $\mathrm{cm}^{-3}$ obtained by Aller (1966) from the observed Balmer lines, although it is less than the value of $3 \times 10^{6}$ electrons $\mathrm{cm}^{-3}$ derived by Rodgers and Searle (1967) from the hydrogen radiation and the forbidden lines of $\mathrm{N}$ II and $\mathrm{S}$ II.

It is a pleasure to thank Dr. W. Sargent and E. Becklin for many discussions, Dr. F. Low for providing us with his 20- $\mu$ magnitudes, and Dr. Ulrich Haug for making $U B V$ measurements of $\eta$ Car. We again thank Dr. Victor Blanco, the director of the Cerro Tololo Inter-American Observatory, and all of his staff for their unfailing hospitality and assistance. We specially thank the extremely capable night assistants Messrs. Carlos Bolelli, Edgardo Figeroa, Ricardo Gonzales, and Patricia Zamorano for their help in making these observations, and Messrs. Duane Ludden and Frank Guzzoni for their operational support.

\section{REFERENCES}

Aller, L. H. 1966, Proc. Nat. Acad. Sci., 55, 671.

Beard, M., and Kerr, F. J. 1967, Australian J. Phys., 19, 875.

Becklin, E. E. 1968 (private communication).

Faulkner, D. J. 1963, Pub. A.S.P., 75, 269.

Feinstein, A. 1963, Pub. A.S.P., 75, 492.

Gratton, L. 1963, in Stellar Evolution, ed. L. Gratton; International School of Physics, Enrico Fermi, Course 28 (New York: Academic Press), p. 297.

Haug, U. 1968 (private communication).

Johnson, H. L. 1964, Bol. Obs. Tonantzintla y Tacubaya, 3, 305.

Low, F. J. 1968 (private communication).

Low, F. J., and Johnson, H. L. 1964, Ap. J., 139, 1130.

McCray, R. 1967, Ap.J., 147, 544.

Neugebauer, G., and Westphal, J. A. 1968, Ap. J. (Letters), 152, L89.

Pagel, B. E. J. 1969, Nature, 221, 325.

Rodgers, A. W., and Searle, L. 1967, M.N.R.A.S., 135, 99.

Seaton, M. J. 1960, Rept. Progr. Phys., 23, 313.

Walker, G. 1967 (private communication). 